\title{
On application of asymmetric Kan-like exact equilibria to the Earth magnetotail modeling
}

\author{
Daniil B. Korovinskiy ${ }^{1}$, Darya I. Kubyshkina ${ }^{1}$, Vladimir S. Semenov ${ }^{2}$, Marina V. Kubyshkina ${ }^{2}$, Nikolai V. Erkaev ${ }^{3,4}$, \\ and Stefan A. Kiehas ${ }^{1}$ \\ ${ }^{1}$ Space Research Institute, Austrian Academy of Sciences, Graz, Austria \\ ${ }^{2}$ Earth's Physics Department, Saint Petersburg State University, St. Petersburg, Russia \\ ${ }^{3}$ Institute of Computational Modelling, FRC "Krasnoyarsk Science Center" SBRAS, Krasnoyarsk, Russia \\ ${ }^{4}$ The Applied Mechanics Department, Siberian Federal University, Krasnoyarsk, Russia
}

Correspondence: Daniil B. Korovinskiy (daniil.korovinskiy@oeaw.ac.at)

Received: 2 January 2018 - Discussion started: 17 January 2018

Accepted: 22 March 2018 - Published: 19 April 2018

\begin{abstract}
A specific class of solutions of the VlasovMaxwell equations, developed by means of generalization of the well-known Harris-Fadeev-Kan-Manankova family of exact two-dimensional equilibria, is studied. The examined model reproduces the current sheet bending and shifting in the vertical plane, arising from the Earth dipole tilting and the solar wind nonradial propagation. The generalized model allows magnetic configurations with equatorial magnetic fields decreasing in a tailward direction as slow as $1 / x$, contrary to the original Kan model $\left(1 / x^{3}\right)$; magnetic configurations with a single $\mathrm{X}$ point are also available. The analytical solution is compared with the empirical T96 model in terms of the magnetic flux tube volume. It is found that parameters of the analytical model may be adjusted to fit a wide range of averaged magnetotail configurations. The best agreement between analytical and empirical models is obtained for the midtail at distances beyond $10-15 R_{\mathrm{E}}$ at high levels of magnetospheric activity. The essential model parameters (current sheet scale, current density) are compared to Cluster data of magnetotail crossings. The best match of parameters is found for single-peaked current sheets with medium values of number density, proton temperature and drift velocity.
\end{abstract}

\section{Introduction}

Studies of magnetosphere dynamics, including substorm events, require a relevant current sheet (CS) stability analysis. This in turn requires a proper choice of the back- ground magnetoplasma configuration. In applications to collisionless plasma, the background equilibrium is to be derived from a solution of the kinetic Vlasov-Maxwell equations. A number of such solutions are derived both numerically (e.g., Burkhart et al., 1992; Pritchett and Coroniti, 1992; Cargill et al., 1994, and others) and analytically (e.g., Schindler and Birn, 2002; Yoon and Lui, 2005; Sitnov and Merkin, 2016; Vinogradov et al., 2016). All these solutions describe symmetric planar current sheets; the only approximate equilibrium solution for bent CS was introduced in the paper of Panov et al. (2012), where the authors present an analysis of direct THEMIS and GOES observations of plasma sheet evolution near substorm onset. Panov et al. (2012) have found the CS bending to be a source of the tailward growing normal magnetic field component $B_{z}$ (in the present paper we use the reference system with $x$ axis pointing tailward, $y$ axis pointing dawnward and $z$ axis pointing north). Hence, bending of the current sheet turns out to be an important parameter for the sheet stability, controlled by the sign of the derivative $\partial B_{z} / \partial x$ (e.g., Hau et al., 1989; Erkaev et al., 2007, 2009; Pritchett and Coroniti, 2010) in many instances.

This result is in line with previous findings revealing that the configuration asymmetry can be an important factor of magnetosphere dynamics. Particularly, Kivelson and Hughes (1990) have first suggested that the CS bending may drop down the reconnection onset threshold. This idea was confirmed later, when Partamies et al. (2009) noticed the seasonal variations in the number of substorm events with maxi- 
mums in winter and summer periods, when dipole tilt angle is bigger (the known geomagnetic activity maximums, e.g., in the $\mathrm{Kp}$ index, are registered contrary around the equinoxes).

Later, this effect was investigated in detail in the paper of Kubyshkina et al. (2015), where it was shown that the substorm probability is higher for about $10-25 \%$ during the periods with tilt angle $>15^{\circ}$, compared to the periods with smaller tilt angles. The direction of the solar wind (SW) flow also affects the substorm probability: it grows for 10-20\% when SW flow direction forces the CS tilt to increase. The statistical analysis has shown that the average substorm intensity (defined by AL value during the event) is lower for larger effective tilts (dipole tilt angle plus solar wind flow inclination). In other words, a large number of weak substorms occur in those time intervals in which effective tilt angles are high, and a smaller number of more intense substorms is observed when tilt angles are small. This also agrees with the results of Nowada et al. (2009), where both AL and AU indices were analyzed for the intervals of negative interplanetary magnetic field $B_{z}$.

In Kubyshkina et al. (2015), the dependence of magnetotail lobe magnetic field (as a proxy of the magnetic flux) on the dipole tilt angle was studied by means of empirical modeling. The average lobe field was found to be smaller for all radial distances in a case of nonzero tilt angles. The decrease reached $10-20 \%$ for maximum tilt angle. This result is reasonable under the assumption that substorm onsets require a lower energy input during the periods of increased dipole tilt. Next, in the paper of Semenov et al. (2015) it was found that there is a clear dependence of the substorm probability on the jumps of the $z$ component of the SW velocity (asymmetric factor), while the jumps of number density or plasma pressure (symmetric factor) turn out to be noneffective. Finally, we should note that the Earth's dipole tilt angle undergoes daily and seasonal variations in the interval of about $\pm 35^{\circ}$, so that it is equal to zero twice a day within about 4 months a year, and during the other 8 months it is never zero. In addition, the solar wind flow direction varies for about $\pm 6^{\circ}$. These variations produce CS inclination, bending and shift from the ecliptic plane. Therefore, the simplest solar wind-magnetosphere configuration (vertical dipole, planar CS, radial solar wind) adopted by the majority of models, is rather untypical and the development of the relevant bent CS models is in high demand.

The first exact solution for two-dimensional (2-D) equilibrium bent CS with nonzero dipole tilt was presented in short notes of Semenov et al. (2015). This solution generalizes the well-known Harris-Fadeev-Kan-Manankova equilibria family (see Yoon and Lui, 2005). In the present paper we investigate the obtained solution to estimate its relevance for the magnetotail CS modeling and stability analysis. For this end, we compare the analytical solution with the empirical Tsyganenko (1995) T96 model and define the analytical model parameters, providing the best agreement.
The paper is organized as follows. In Sect. 2 we describe the analytical solution for bent CS. In Sect. 3 we compare analytical and empirical T96 solutions. In Sect. 4 we present the further generalization of the analytical model, providing more realistic profiles of $B_{z}$ in the equatorial plane. Then, the model-typical scales are compared with in situ data. Discussion and conclusions finalize the paper in Sect. 5.

\section{Analytical solution}

For two-component (proton + electron) isothermal plasma with Maxwellian distribution functions and constant current velocity the system of Vlasov-Maxwell equations can be reduced to the 2-D Grad-Shafranov equation (see Schindler, 1972; Yoon and Lui, 2005) for the dimensionless magnetic potential $\Psi=(0, \Psi, 0)$,

$\frac{\partial^{2} \Psi}{\partial x^{2}}+\frac{\partial^{2} \Psi}{\partial z^{2}}=e^{-2 \Psi}$.

The quantity $\Psi$ is normalized for $\left(-B_{0} L\right)$, where $L=$ $2 c T_{i} /\left(e B_{0} V_{i}\right)$ is the typical scale of CS in the normal direction, $B_{0}=\sqrt{8 \pi n_{0}\left(T_{e}+T_{i}\right)}$ is the lobe magnetic field, $n_{0}=n_{0 e}=n_{0 i}$ is the typical number density, $T_{e, i}$ are the electron and ion temperatures, respectively, and $V_{e, i}$ are the corresponding drift velocities, fulfilling the condition

$V_{i} / T_{i}+V_{e} / T_{e}=0$.

Equation (2) expresses the condition of the zero electrostatic potential. The model of an ion-dominated CS, where $\left|V_{i} / V_{e}\right|>T_{i} / T_{e}$, is considered in the paper of Yoon and Lui (2004). In the case of Maxwellian distribution functions condition (2) can be satisfied by means of the proper choice of the reference system, while in the general case of non-Maxwellian distribution functions it cannot be fulfilled (Schindler and Birn, 2002).

A series of analytical solutions of Eq. (1) was found by Walker (1915), who showed that the solution may be expressed via an arbitrary generating function $g$ of the complex variable $\zeta=x+i z$,

$$
\begin{aligned}
& e^{-2 \Psi}=\frac{4\left|g^{\prime}\right|^{2}}{\left(1+|g|^{2}\right)^{2}}, \\
& g^{\prime}=\frac{\mathrm{d} g(\zeta)}{\mathrm{d} \zeta} .
\end{aligned}
$$

With the solution (3), the equilibrium magnetoplasma configuration takes the form

$$
\begin{aligned}
\Psi & =\ln \left(\frac{1+|g|^{2}}{2\left|g^{\prime}\right|}\right), \\
n & =\exp (-2 \Psi), \quad p=0.5 \exp (-2 \Psi),
\end{aligned}
$$

where $p$ is the plasma pressure. By definition, the dimensionless magnetic field components are $B_{x}=-\partial \Psi / \partial z$ and $B_{z}=+\partial \Psi / \partial x$. 
The particular choice of the generating function $g$ specifies the particular CS model. In the current paper we consider the family of Harris-like models, including the classical Harris (1962) current sheet, the Fadeev et al. (1965) solution (Harris sheet complemented by an infinite chain of magnetic islands along the neutral plane), the Kan (1973) solution (Harris sheet with quasi-dipole), and the Manankova et al. (2000) solution, representing the combination of all previous models. The last one is specified by the generating function

$g(\zeta)=f+\sqrt{1+f^{2}} \exp \left[i\left(\zeta-\frac{b}{\zeta-a}\right)\right]$.

Solution (6) contains three real parameters $a, b$ and $f$, where $a$ specifies the shift along the $x$ axis, $b$ controls the field line elongation, and $f$ defines the current density in the magnetic islands. Generating functions for other listed models are the special cases of the function (6). Namely, one should set $(f=0, a \neq 0, b \neq 0)$ for the Kan solution; $(f \neq 0, a=$ $b=0)$ for the Fadeev solution, and $(f=a=b=0)$ for the Harris solution.

The solution for a bent CS is developed in the paper of Semenov et al. (2015) by substituting the complex parameters $a \rightarrow i a$ and $b \rightarrow b_{0} e^{i \varphi}$ in Eq. (6). The complex parameter $a$ controls the shift of the CS in the $z$ direction and $\varphi$ controls the dipole tilt angle. For the case of bent CS without plasmoids (Kan-like model, $f=0$ ) the solution (6) takes relatively simple form,

$\Psi=\ln \left(\frac{\cosh Z_{*}}{\sqrt{W}}\right)$,

$Z_{*}=z-\frac{b_{0} x \sin (\varphi)-b_{0}(z-a) \cos (\varphi)}{R^{2}}$,

$W=$

$1+\frac{b_{0}^{2}+2 b_{0}\left(x^{2}-(z-a)^{2}\right) \cos (\varphi)+4 b_{0} x(z-a) \sin (\varphi)}{R^{4}}$,

where $R^{2}=x^{2}+(z-a)^{2}$. Configurations of this type possess a dipole singularity at $(x, z)=(0, a)$ and two additional singularities at $(x, z)=\left( \pm \sqrt{b_{0}} \sin (\varphi / 2), a \mp \sqrt{b_{0}} \cos (\varphi / 2)\right)$, rotating twice as slow as a dipole does. Hence, the effective dipole tilt is equal to $\varphi / 2$. For positive tilt angles the CS is bent and uplifted over the ecliptic plane, and for negative tilts the CS is shifted down.

The set of magnetic configurations for dipole tilt angle $\mathrm{PHI}=\{0,30,60,120\}$ degrees clockwise $(\mathrm{PHI}=-\varphi / 2)$ is shown in Fig. 1. The two first cases $\left(0\right.$ and $\left.30^{\circ}\right)$ can be observed in the Earth's magnetosphere, and other cases are shown here to illustrate the model behavior. White asterisks in Fig. $1 \mathrm{c}$ and $\mathrm{d}$ mark the $\mathrm{X}$ points $\left(B_{x}=B_{z}=0\right)$, being an attribute of the Kan-like solution. In the symmetric Kan model the $\mathrm{X}$ point is located at infinity, but in bent sheets it starts to approach the dipole with increasing tilt angle. This $\mathrm{X}$ point is not produced by magnetic reconnection, and it does not break a steady state equilibrium of the CS. However,
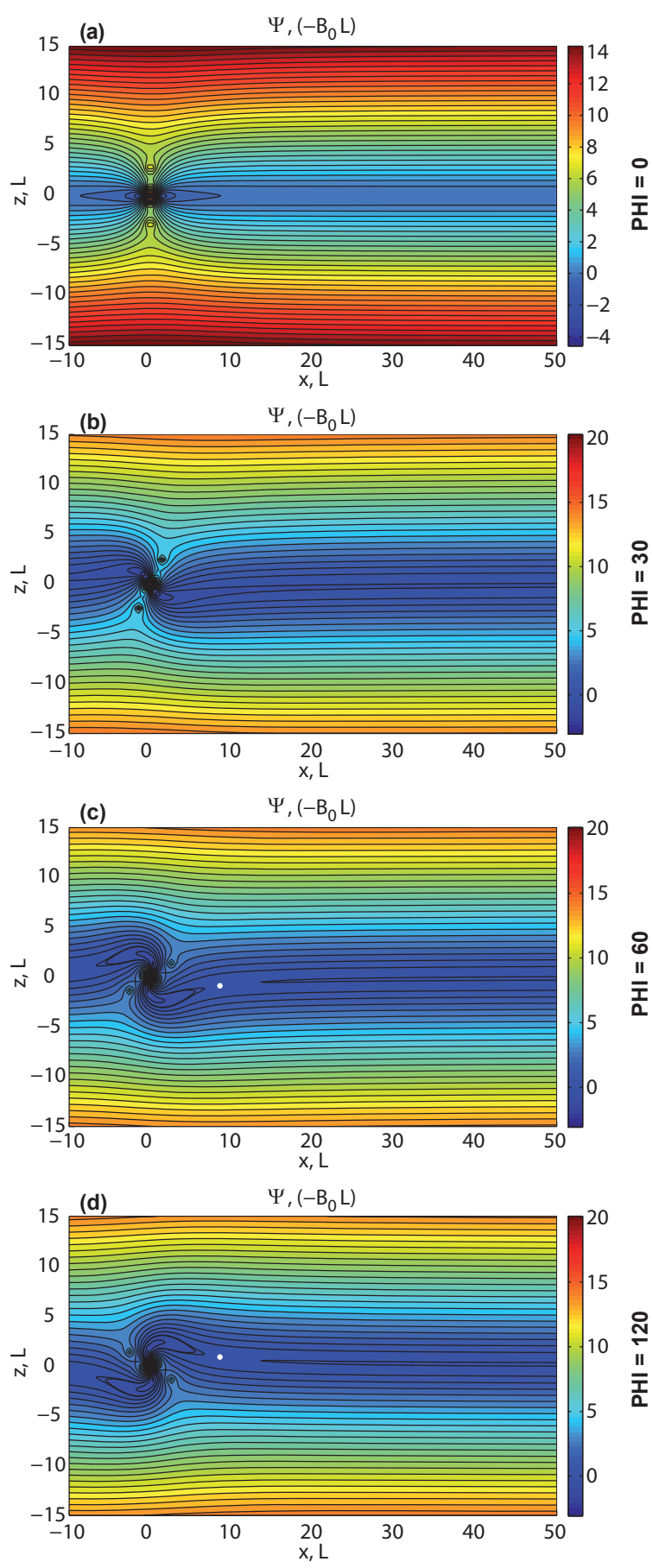

Figure 1. Magnetic potential $\Psi(x, z)$, calculated from the asymmetric Kan model (Eqs. 7-9) with parameters $a=0$ and $b_{0}=8$. Solutions with dipole tilt angles $\mathrm{PHI}=\left\{0^{\circ}, 30^{\circ}, 60^{\circ}, 120^{\circ}\right\}$ clockwise are plotted on panels (a)-(d), respectively. $\mathrm{PHI}=-\varphi / 2$ of the analytical model. Spatial units are normalized for typical CS width $L$. Magnetic potential is normalized for $\left(-B_{0} L\right)$. X points are marked white.

the appearance of the $\mathrm{X}$ point can be considered as a manifestation of potentially unstable configuration. In such a case, 


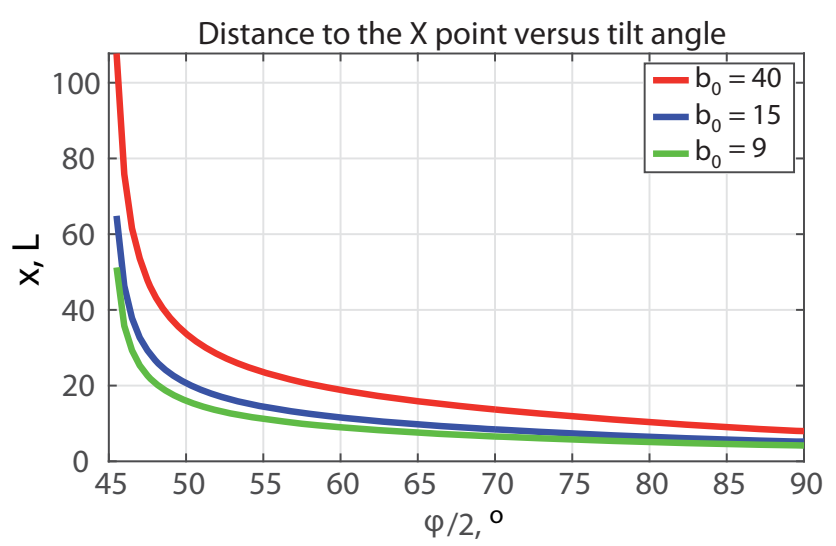

Figure 2. X-point location vs. the effective tilt angle $\varphi / 2$ in the asymmetric Kan solution (Eqs. 7-9) with $a=0$ and parameters $b_{0}=40$ (red), $b_{0}=15$ (blue), and $b_{0}=9$ (green).

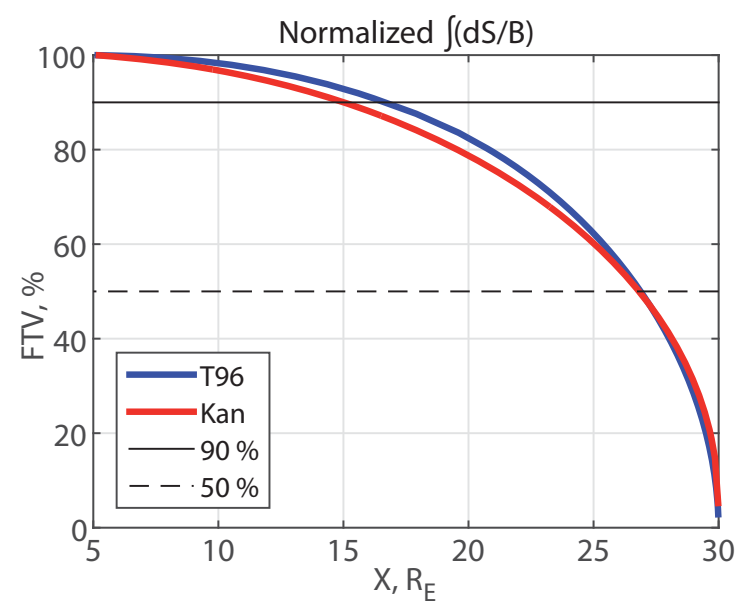

Figure 3. Flux tube volume $\int_{(30,-2.4)}^{(x, z)}(\mathrm{d} S / B)$ normalized to the full FTV $\int_{(30,-2.4)}^{(5,1.5)}(\mathrm{d} S / B)$, in percent, calculated by T96 model (blue) and by the Kan model (red) for the quiet conditions with tilt angle $\mathrm{PHI}=30^{\circ}$ clockwise (model parameter $\varphi=-60$ ). Other model parameters are given in the legend of Fig. 4b. The Earth is on the left.

the X-point motion towards the dipole with increasing tilt angle could mean that CS evolves toward an unstable state. According to the solution (Eqs. 7-9), the X-point location also depends on the CS width $L$ and model parameter $b_{0}$. The Xpoint position as a function of $\varphi$ is plotted in Fig. 2 for three values of $b_{0}$, corresponding to three different levels of geomagnetic activity (see Fig. 4, right column). It is seen that for tilt angles $|\varphi / 2|<45^{\circ}$ the $\mathrm{X}$ point stays very far beyond $60 R_{\mathrm{E}}$ for any realistic value of $L$ and $b_{0}$, e.g., for $b_{0}=8$ (the value, corresponding to quiet magnetotail) and $|\varphi / 2|=45^{\circ}$ the $\mathrm{X}$ point stays as far as $\approx 340 \mathrm{~L}$. For $|\varphi / 2|=60^{\circ}$ (almost 2 times more than the Earth maximal dipole tilt) an approach to $8.5 \mathrm{~L}$ is achieved.

\section{Comparison with the T96 model}

Topologically, magnetic configurations plotted in Fig. 1 are very similar to that of the Earth's magnetosphere. However, to estimate the relevance of the analytical solution one should compare some important numerical characteristics of the CS model with the corresponding values registered in real observations. This can be done by utilizing empirical magnetic field models, providing realistic averaged magnetospheric configurations at various levels of magnetospheric activity. Of course, we should keep in mind that the real magnetosphere is an essentially three-dimensional structure. Following the dipole tilt (and solar wind flow direction) variations, the magnetotail CS bends and shifts from the equatorial plane in the $z$ direction (at most $\sim 3 R_{\mathrm{E}}$ for maximum tilt) and also warps in the $y$ direction. These effects are well pronounced in empirical magnetospheric models, but the 2-D analytical model is evidently unable to reproduce all these complex deformations. Therefore, we restrict our study to the noonmidnight plane $y=0$, and the two main effects manifested in that plane: CS bending and shifting in $z$ direction.

To explore the appropriateness of the here presented analytical solution for bent CS, we compare the predicted magnetic flux tube volume (a proxy for the entropy) with that calculated from the empirical model of Tsyganenko (1995) T96. We consider the flux tube volume (FTV) instead of the entropy, since the analytical solution is isothermal. This quantity is chosen due to its importance for the magnetotail dynamics. As was claimed by Birn et al. (2009) and verified by in situ data analysis (Sergeev et al., 2014), any bursty bulk flow (BBF), produced by reconnection in the magnetotail and moving toward the Earth, stops near that particular point where the entropy of the ambient plasma is equal to that inside the BBF. The distribution of entropy along the magnetotail is also an important factor for the stability analysis (Birn et al., 2009) and for the study of wave (oscillation) generation and dissipation (Panov et al., 2016).

The FTV is determined in the same way for both analytical and empirical models: we integrate $\mathrm{d} S / B$ along magnetic field lines, where $\mathrm{d} S$ is the field line length element and $B=\sqrt{B_{x}^{2}+B_{z}^{2}}$. In the T96 model the location of the flux tube is computed by means of field line tracing; in the analytical model this is a curve of constant $\Psi$. As one of the first steps, the values of FTV of a single flux tube are compared. The model parameters correspond to the quiet magnetospheric conditions with tilt angle of $30^{\circ}$ clockwise (see the legend of Fig. 4b). FTVs are calculated along the magnetic field line with a node at $(x, z)=(30,-2.4)$. To eliminate singularities, we excluded the near-Earth region $x<5 R_{\mathrm{E}}$, so that the total FTVs are calculated as $\int_{(30,-2.4)}^{(5,1.5)} \mathrm{d} S / B$. The results are shown in Fig. 3 by the blue curve for T96 and by the red curve for the Kan model. The values of FTV, normalized for total FTV, are plotted as a function of $x$. It is seen that two models demonstrate rather close results. A total of 
$\mathrm{PHI}=0^{0}$
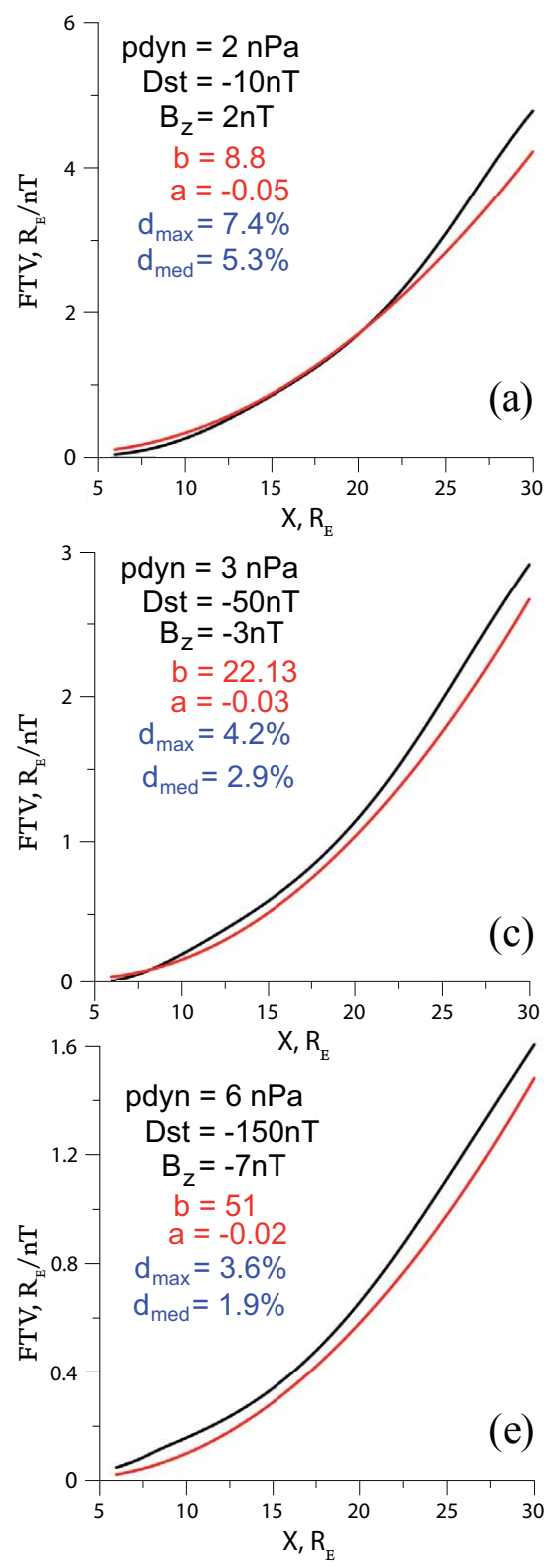

$\mathrm{PHI}=30^{\circ}$
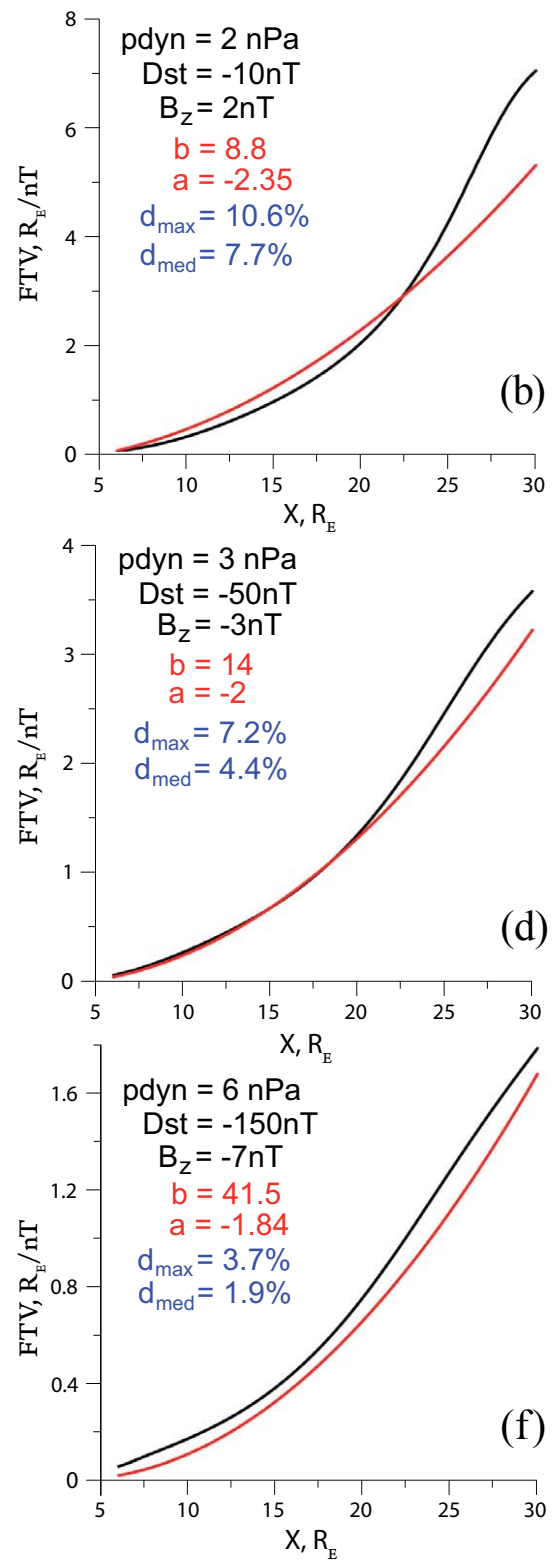

Figure 4. Flux tube volumes: analytical solution (red curves) and T96 (black curves) for quiet (a, b), substorm (c, d) and storm (e, f) conditions are plotted for tilt angles $0^{\circ}(\mathbf{a}, \mathbf{c}, \mathbf{e})$ and $\mathrm{PHI}=30^{\circ}$ clockwise $(\mathbf{b}, \mathbf{d}, \mathbf{f})$. Input parameters for the T96 model (black text), for the Kan-like model (red text), and SD normalized for average FTV (blue text) are given in legends. The Earth is on the left.

$90 \%$ of FTV are provided by the farther half of the tube, $x \in[15,30] R_{\mathrm{E}}$, and $50 \%$ of FTV are concentrated in the most distant interval within $3-4 R_{\mathrm{E}}$ in the $x$ direction from the tube node.

Then, FTVs, calculated by means of analytical and empirical models, are compared at different levels of magnetospheric activity, characterized by input parameters of the T96 model (Dst index, the SW dynamical pressure, $p_{\text {dyn }}$, and SW magnetic field components $B_{y}^{\mathrm{sw}}$ and $\left.B_{z}^{\mathrm{sw}}\right)$. Three sets of parameters are taken to specify the quiet magnetotail $\left\{\right.$ Dst $\left.=-10, p_{\text {dyn }}=2 \mathrm{nPa}, B_{z}^{\mathrm{sw}}=2 \mathrm{nT}\right\}$, substorm conditions $\left\{\mathrm{Dst}=-50, p_{\mathrm{dyn}}=3 \mathrm{nPa}, B_{z}^{\mathrm{sw}}=-3 \mathrm{nT}\right\}$ and storm $\left\{\right.$ Dst $\left.=-150, p_{\text {dyn }}=6 \mathrm{nPa}, B_{z}^{\mathrm{sw}}=-7 \mathrm{nT}\right\}$. Magnetic field component $B_{y}^{\mathrm{sw}}$ was set equal to zero. Parameters $a$ and $b_{0}$ of the analytical solution (7-9) are found numerically to minimize the SD between two models. The results (FTV vs. $x$ coordinate of the flux tube node) are presented in Fig. 4, where red lines plot analytical solutions and black ones plot the T96 

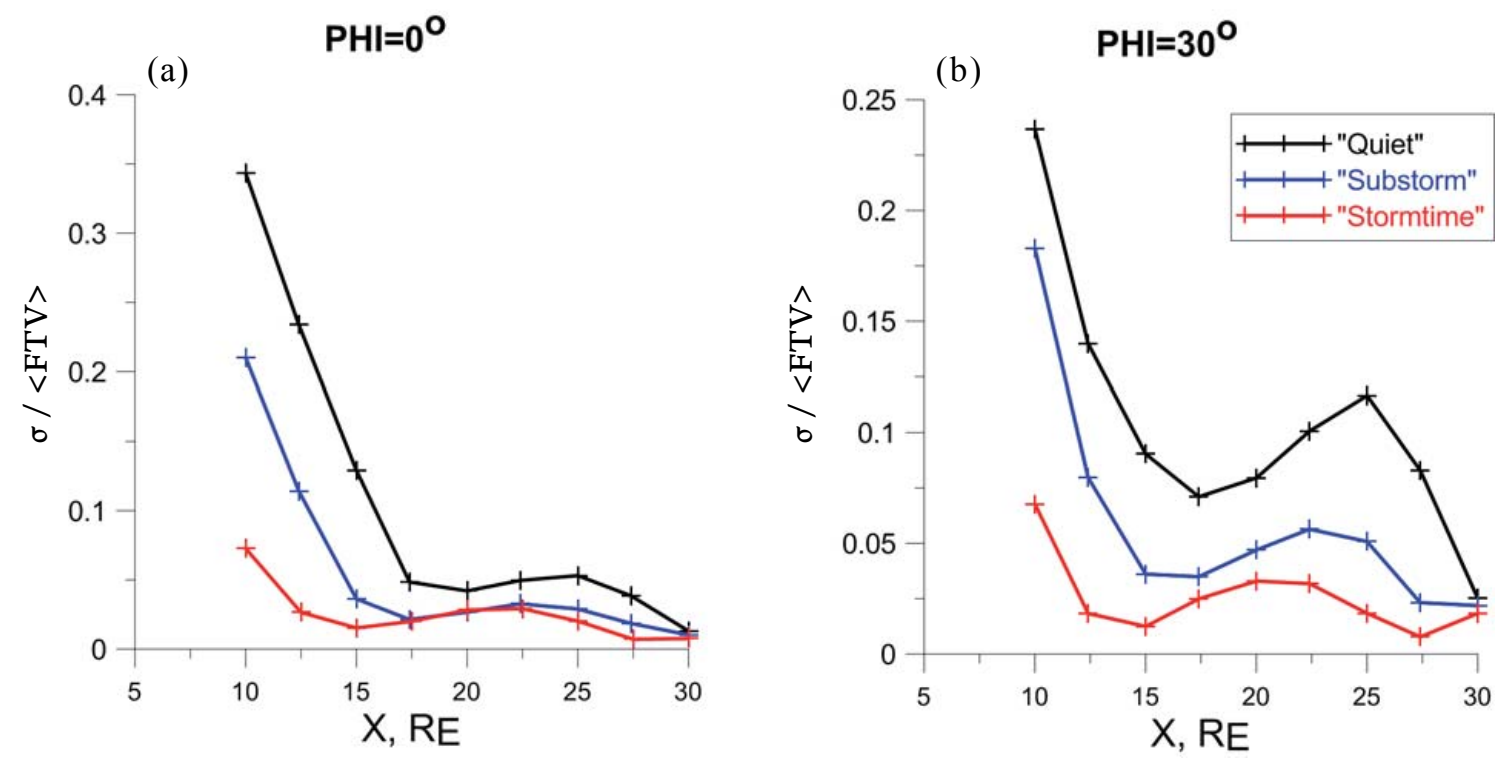

Figure 5. Comparison of analytical and empirical models for symmetric (dipole tilt $0^{\circ}$, a) and bent $\left(30^{\circ}\right.$ clockwise, b) current sheets Standard deviations, $\sigma$, normalized for average FTV, are shown as functions of $x_{0}$, where $x_{0}$ is the center of the region under consideration $\left[x_{0}-2.5, x_{0}+2.5\right]$, for quiet (black curves), substorm (blue curves) and storm (red curves) conditions. The Earth is on the left.

results. The left column shows the symmetrical case (zero dipole tilt), and the right column corresponds to the dipole tilt angle of $30^{\circ}$ clockwise.

One can see that the agreement between two models is quite good, with the maximal SDs varying within $2-11 \%$. The values of $d_{\max }=\sigma_{\max } /\langle\mathrm{FTV}\rangle$ are given in legends of Fig. 4, where $\sigma$ is the SD and $\langle\mathrm{FTV}\rangle$ is the average FTV. The better agreement is achieved for disturbed magnetospheric conditions, i.e., the analytical model describes the stretched CSs even better than the thicker ones. It is found that minimal difference between two models is obtained when parameter $a$ is very close to the medium neutral sheet position determined from the empirical model. The best-fit value of the parameter $b_{0}$, controlling the field lines stretching and the CS thinning, depends on the level of activity and the dipole tilt angle. It grows from 8.8 for the quiet magnetosphere to 51 for storm conditions. At any fixed distance, the stretching of field lines makes the FTV decrease with growing magnetospheric activity. For example, at the distance of $x=30 R_{\mathrm{E}}$ it changes from $\approx 5 R_{\mathrm{E}} / n T$ for "quiet" conditions to $\approx 3 R_{\mathrm{E}} / n T$ for "substorm" conditions and to the $\approx 1.6 R_{\mathrm{E}} / n T$ for "storm-time" conditions. On the contrary, the asymmetric deformation of CS (dipole tilt angle) forces the FTV to increase.

Figure 4 shows a comparison of two models within the large interval $x \in[5,30]$. To detect the best-matching region we performed the same analysis for eight short overlapping intervals $x \in[7.5,12.5]+2.5 n$, where $n=0,1, \ldots, 8$. The normalized SD as a function of $x_{0 n}$, where $x_{0 n}$ is the center of corresponding interval, is shown in Fig. 5, where three features are observed: (1) SD grows toward the Earth and exceeds $10 \%$ for $x<15 R_{\mathrm{E}}$ for all activity levels, (2) devi- ations are bigger for the more quiet magnetosphere environment, and (3) deviations are smaller for a tilt angle of $30^{\circ}$. Compared to results of the large interval analysis (Fig. 4), dependence on the activity level is the same, and dependence on the tilt angle demonstrates opposite behavior. Overall, analytical and empirical models show good agreement beyond $15 R_{\mathrm{E}}$, improving with growing activity.

\section{Normal magnetic component and current density}

The results of the previous section show that parameters of the asymmetric Kan-like model may be adapted to provide rather good agreement with the magnetotail CS, especially in a distant tail beyond $15-20 R_{\mathrm{E}}$, and especially for bent current sheets. However, until now the practical usage of this model encountered a substantial obstacle, related to the behavior of the normal magnetic field component. It can be easily checked that in the distant tail the Kan model yields $B_{z} \sim 1 / x^{3}$, while in reality $B_{z}$ decreases as $1 / x$ or even slower (e.g., Behannon and Ness, 1966; Mihalov et al., 1968; Behannon, 1970; Wang and Lyons, 2004; Yue et al., 2013). For plane and axially symmetric current sheets the solution with $B_{z} \sim 1 / x^{\alpha}$ with arbitrary $\alpha$ is found in Vasko et al. (2013). For Kan-like models considered in the current paper the $B_{z}$ problem may be solved by introducing one more parameter in the generating function $g(\zeta)$. With the additional parameter $n$, general asymmetric model takes the form (compare to Eq. 16 of Yoon and Lui, 2005)

$g(\zeta)=f+\sqrt{1+f^{2}} \exp \left[i\left(\zeta^{n}-\frac{b}{(\zeta-a)^{k}}\right)\right]$. 

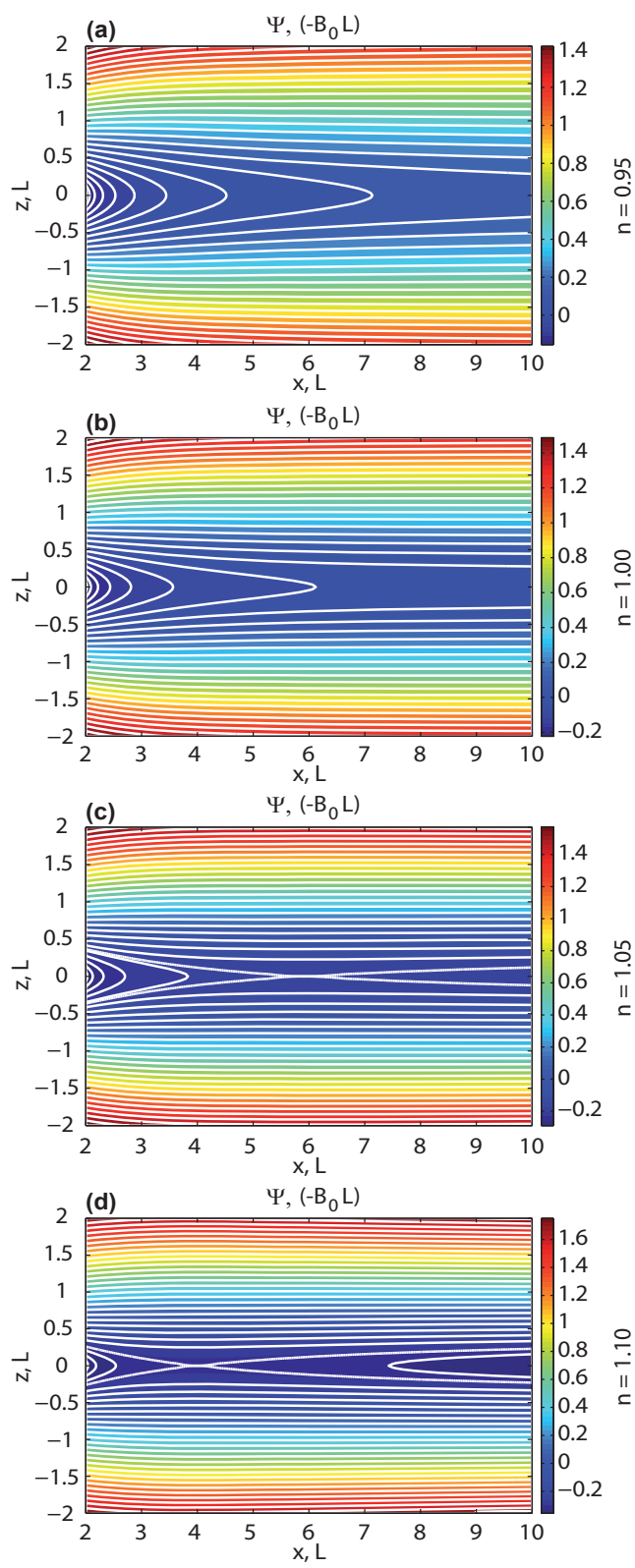

Figure 6. The values of magnetic potential $\Psi(x, z)$, calculated from analytical model (Eqs. 11-16), are shown by color for model parameter $n=\{0.95,1,1.05,1.1\}$ on panels (a)-(d), respectively. Other parameters $\left\{a=0, b_{0}=1, \varphi=0, f=0, k=1\right\}$ are the same. Magnetic field lines are plotted by white curves. Panel (b) shows the original Kan solution.

Assuming $\{f, n, k\}$ to be real values, $a=a_{1}+i a_{2}$, and $b=$ $b_{0} \exp (i \varphi)$, we derive

$$
\begin{aligned}
& \Psi=\ln \left(\frac{f \cos X_{*}+\sqrt{1+f^{2}} \cosh Z_{*}}{\sqrt{W}}\right), \\
& X_{*}=r^{n} \cos (n \vartheta)-\frac{b_{0}}{R^{k}} \cos (k \Theta-\varphi),
\end{aligned}
$$

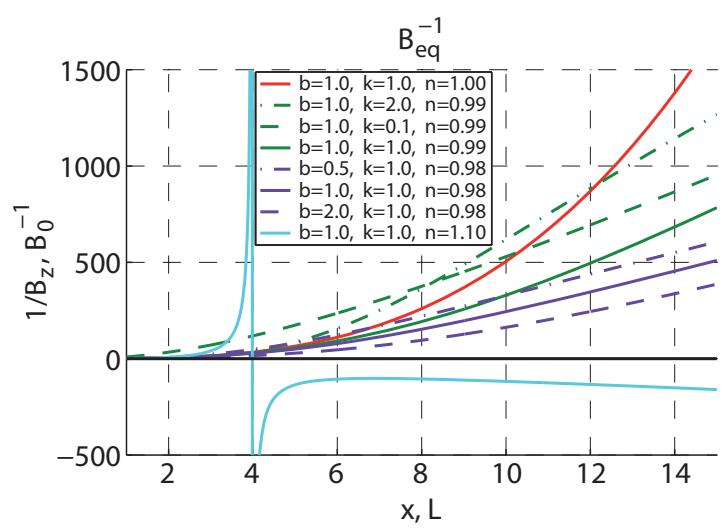

Figure 7. Profiles $1 / B_{z}(x, 0)$ for symmetric Kan-like CS, calculated from an analytical model (Eqs. 11-16). Parameters $\left\{a_{1}=\right.$ $\left.0, a_{2}=0, \varphi=0, f=0\right\}$ are the same. Other parameters are $\left\{b_{0}=\right.$ $1, k=1, n=1\}$ (red), $\left\{b_{0}=1, k=2, n=0.99\right\}$ (dark-green dashdotted), $\left\{b_{0}=1, k=0.1, n=0.99\right\}$ (dark-green dashed), $\left\{b_{0}=\right.$ $1, k=1, n=0.99\}$ (dark-green solid), $\left\{b_{0}=0.5, k=1, n=0.98\right\}$ (violet dash-dotted), $\left\{b_{0}=1, k=1, n=0.98\right\}$ (violet solid), $\left\{b_{0}=\right.$ $2, k=1, n=0.98\}$ (violet dashed), and $\left\{b_{0}=1, k=1, n=1.1\right\}$ (cyan). Red curve shows the original Kan solution. Units are normalized for CS typical width $L$ and for $B_{0}^{-1}$.

$$
\begin{aligned}
Z_{*}= & r^{n} \sin (n \vartheta)+\frac{b_{0}}{R^{k}} \sin (k \Theta-\varphi), \\
W= & n^{2} r^{2(n-1)}+\frac{b_{0}^{2} k^{2}}{R^{2(k+1)}}+2 n k b_{0} \frac{r^{n-1}}{R^{k+1}} \cos [(n-1) \vartheta \\
& +(k+1) \Theta-\varphi], \quad \vartheta=\arctan \left(\frac{z}{x}\right), \\
r= & \sqrt{x^{2}+z^{2}}, \quad \\
R= & \sqrt{\left(x-a_{1}\right)^{2}+\left(z-a_{2}\right)^{2}}, \quad \theta=\arctan \left(\frac{z-a_{2}}{x-a_{1}}\right) .
\end{aligned}
$$

For symmetric Kan-like CS without plasmoids $(a=0, f=$ $0, \varphi=0)$, the quantity $B_{z}$ at the $x$ axis takes the simple form $B_{z}(x, 0)=-(\partial W / \partial x) /(2 W)$. It is seen that the Kan solution $(n=1)$ is the only degenerated case when the first term of $W$ turns to 1 and its derivative to zero; hence, in the distant tail $B_{z} \sim\left(1 / x^{2+k}\right)$ due to the rightmost term of expression (14). For any $n \neq 1$ we have $(\partial W / \partial x) / W \rightarrow O(1 / x)$.

Parameter $n$ controls flaring of magnetic field lines; values of $n>1$ force strong convergence of the CS field lines toward the $x$ axis, and hence the location of the $\mathrm{X}$-line is drastically dependent on $n$. This feature is illustrated in Fig. 6, where four symmetric magnetic configurations with $(a=$ $\left.0, \varphi=0, f=0, b_{0}=1, k=1\right)$ and $n=\{0.95,1,1.05,1.1\}$ are plotted. In Fig. 7 reverse values of the equatorial magnetic field, $B_{z}^{-1}(x, 0)$, are plotted for several sets of the model parameters. The set of green curves illustrates contribution of the parameter $k$. The set of violet curves shows the effect of the parameter $b_{0}$ variation. The set of solid curves demonstrates the parameters $n$ impact. It is seen that (a) all curves except the red one (original Kan solution, $n=1$ ) tend to 

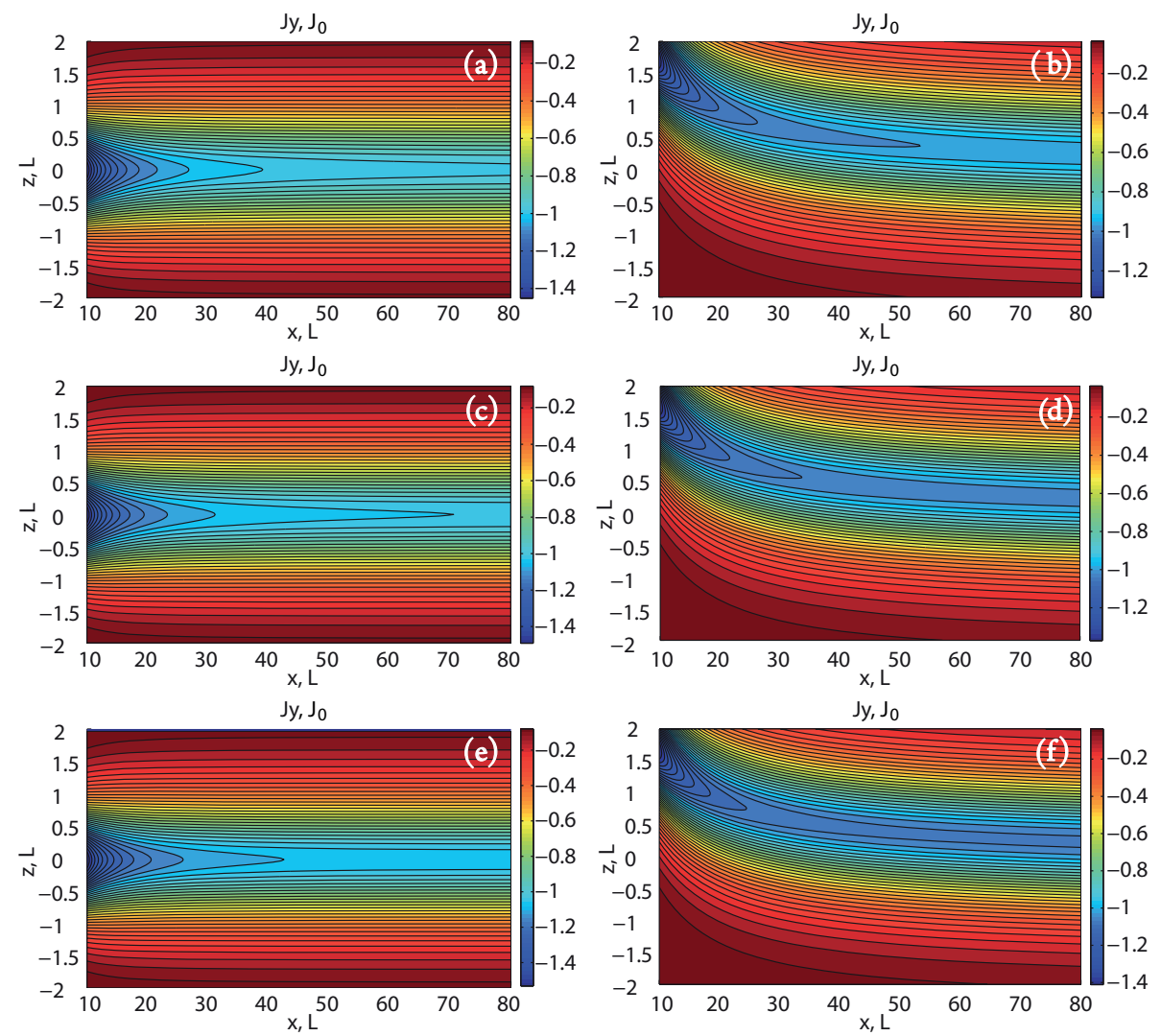

Figure 8. Current density $J_{y}(x, z)$ by an analytical model (Eqs. 11-16) for $\left\{a_{1}=0, a_{2}=-0.03, b=22.13, f=0, k=1\right\}$ is shown for three values of the parameter $n$ for plane sheets $(\mathbf{a}, \mathbf{c}, \mathbf{e}, \varphi=0)$ and curved sheets $(\mathbf{b}, \mathbf{d}, \mathbf{f}, \varphi=60)$. In panels $(\mathbf{a}, \mathbf{b}), n=0.995$. In $(\mathbf{c}, \mathbf{d})$ $n=1$. In (e, f), $n=1.005$. Panel (c) corresponds to the plane substorm sheet (see Fig. 4c). Units are normalized for CS typical width $L$ and $J_{0}=c B_{0} /(4 \pi L)$.

$\mathrm{O}(x)$, and (b) numerical values of $B_{z}$ are highly variable depending on different combinations of parameters $\left\{b_{0}, k, n\right\}$.

In two dimensions, contributions of parameters $n$ and $\varphi$ are shown in the next two plots. Figures 8 and 9 present $J_{y}(x, z)$ and $B_{z}(x, z)$, respectively, for six sets of the model parameters, where parameters $a_{1}=0, a_{2}=-0.03, f=0, b_{0}=$ 22.13, $k=1$ are the same. Panels (a) show the solutions for $(n=0.995, \varphi=0)$. Panels (b) show the solutions for the bent sheet $(n=0.995, \varphi / 2=30)$. On panels (c) solutions for a plane substorm CS model (see Fig. 4c) with $n=1$ and $\varphi=0$ are shown; the bent sheet $(n=1, \varphi / 2=30)$ quantities are plotted on panels (d). On panels (e) parameter $n=1.005$ and $\varphi=0$, and on panels (f) $n=1.005$ and $\varphi / 2=30$.

Figure 8 demonstrates that the CS width is almost uniform on $x$ and is not affected by tilt angle, controlling only the sheet location (vertical shift may be recouped by the proper choice of parameter $a_{2}$ ). With increasing parameter $n$, the sheet is thinning and, correspondingly, the peaking current density is growing. The same effect is produced by enhanced geomagnetic activity. Comparison of current densities for quiet and storm conditions (not shown) reveal 20\% reduc- tion of the CS width and $20 \%$ growth of the peaking current density.

Figure 9 shows that even so weak a variation of parameter $n$ affects the distribution of $B_{z}$, mostly near to the sheet center. The range of appropriate values of $n$ is restricted from above by the solution geometry (X-point location). Say, for current model parameters and with $n=1.01$, the $\mathrm{X}$ point is located at $x \approx 65$ in a plane sheet, and it approaches $x \approx 50$ for $\varphi / 2=30$. As expected, the increase of tilt angle $\varphi$ enhances the value of $B_{z}$, so that for $\varphi / 2=30^{\circ} B_{z}$ is growing 10 times.

The solution (Eqs. 11-16) is written in normalized units, where the magnetic field is normalized for the lobe value $B_{0}$, and normalization constants for the length scale and current density are

$$
\begin{aligned}
& L=\frac{2 c T_{i}}{e B_{0} V_{i}}=2 \cdot 10^{3} \cdot \frac{T_{i}[\mathrm{keV}]}{B_{0}[\mathrm{nTT}] V_{i}\left[\mathrm{~km} \mathrm{~s}^{-1}\right]}, 10^{3} \mathrm{~km}, \\
& J_{0}=\frac{c B_{0}}{4 \pi L}=0.8 \cdot \frac{B_{0}[\mathrm{nT}]}{L\left[10^{3} \mathrm{~km}\right]}, \mathrm{nA} \mathrm{m}^{-2} .
\end{aligned}
$$

To estimate the relevance of this scaling, we make use of Cluster data of magnetotail CS crossings, pre- 

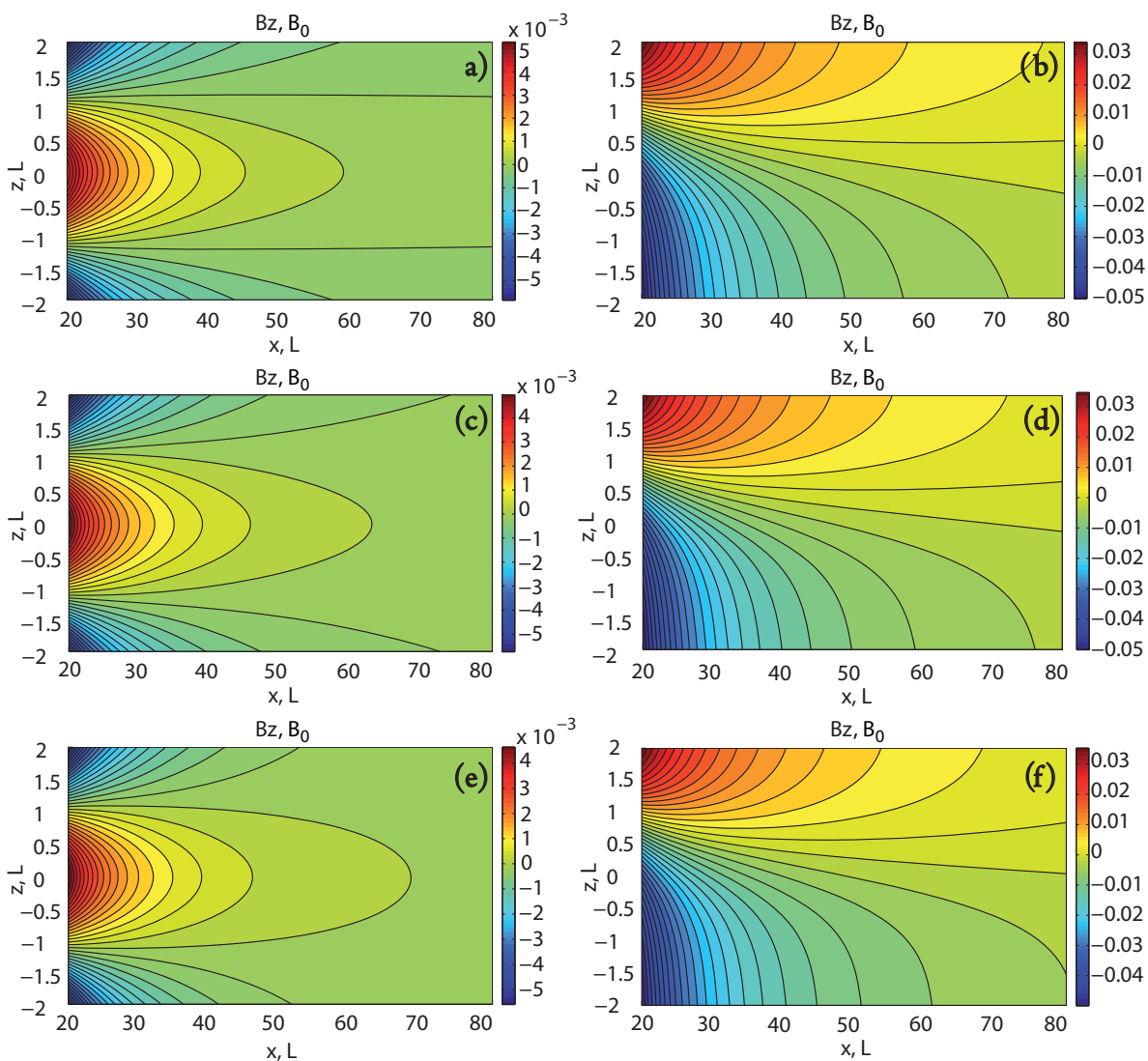

Figure 9. Magnetic field component $B_{z}(x, z)$ by an analytical model (Eqs. 11-16) for $\left\{a_{1}=0, a_{2}=-0.03, b=22.13, f=0, k=1\right\}$ is shown for three values of the parameter $n$ for plane sheets $(\mathbf{a}, \mathbf{c}, \mathbf{e}, \varphi=0)$ and curved sheets $(\mathbf{b}, \mathbf{d}, \mathbf{f}, \varphi=60)$. In panels $(\mathbf{a}, \mathbf{b}), n=0.995$. In $(\mathbf{c}, \mathbf{d}), n=1$. In (e, f) $n=1.005$. Panel (c) corresponds to the plane substorm sheet (see Fig. 4c). Units are normalized for CS typical width $L$ and $B_{0}$.

sented in Table 1 of Runov et al. (2006). Assuming $V_{i}=$ $\sqrt{V_{x}^{2}+V_{y}^{2}+V_{z}^{2}}$ and $B_{0}=B_{L}$, the quantities $L$ and $J_{0}$ are calculated. The plot of $J_{0}(L)$ is shown in Fig. 10. Most of the points, which we call "regular", lie within the interval of $L \in[2,8] 10^{3} \mathrm{~km}$ and $J_{0} \in[3,15] \mathrm{nAm}^{-2}$ (red asterisks). Other points represent extremely small values of CS parameters, such as very low ion temperature $\left(T_{i}<2 \mathrm{keV}\right.$, blue crosses), drift velocity ( $V_{i}<35 \mathrm{~km} \mathrm{~s}^{-1}$, blue diamonds) and number density $\left(n_{i}<0.2 \mathrm{~cm}^{-3}\right.$, blue asterisks). A single case an of extremely high value of $V_{i}=659 \mathrm{~km} \mathrm{~s}^{-1}$ is marked by a magenta circle.

Figure 11 shows the model normalization constant $J_{0}$ vs. peaking observed perpendicular current density (blue curve in Fig. 2 of Runov et al., 2006). It is seen that analytical estimates and measured values of $J_{0}$ mismatch in all extreme cases of Fig. 10. In other cases ("regular" points, red asterisks) the model estimate agrees with observed values with an accuracy up to a coefficient $k \in[0.5,2]$ (except for the cases 4 and 23 of Runov et al., 2006, when the discrepancy increases by 2.5 times). Thus, the best match of current densities is found for cases $\{1-3,5-7,11,15-18,22,25-28\}$, which are mostly single-peaked current sheets. The analytical model (Eqs. 11-16) preserves basic features of the initial Harris solution, and hence it is unable to resolve the complex CS structure, such as bifurcated or embedded current sheets (see, e.g., Hoshino et al., 1996; Nakamura et al., 2006; Runov et al., 2006; Artemyev et al., 2009; Petrukovich et al., 2015). It means that the cross-sheet profiles of current density in our model (not shown) resemble the Harris profiles, shown in Figs. 2 and 3 of Runov et al. (2006). Hence, analytical estimates of the CS width usually exceed the real values. However, in some cases (e.g., cases 3, 27 and 28) the Harris profiles may be more or less relevant to real current sheets.

\section{Discussion and conclusions}

In empirical models (T89, T96, T01, TS05, etc.) magnetic field configurations with any plasma populations are not force-balanced since $\nabla \times[\mathbf{j} \times \mathbf{B}] \neq 0$, or there is no $\nabla P$ to balance Ampere's force (Zaharia et al., 2003). That is why we crucially need kinetic force-balanced CS models for many magnetospheric studies, such as wave generation in 


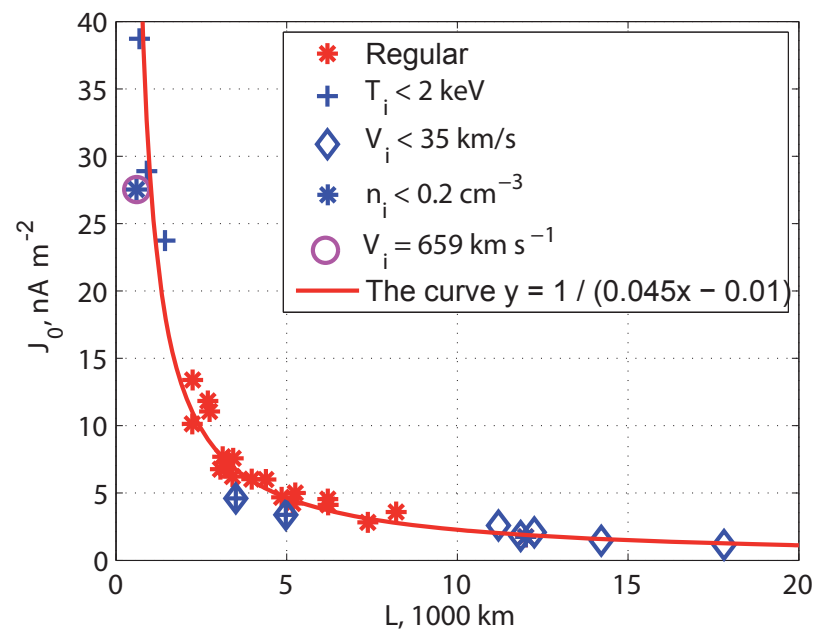

Figure 10. Analytical model units: current density $J_{0}$ vs. spatial scale $L$ from Eqs. (17) and (18) for Cluster data of current sheet crossings, presented in Table 1 of Runov et al. (2006). Blue crosses show cases of the lowest ion temperature, $T_{i}<$ $2 \mathrm{keV}(8,9,12,13,14$ in Table 1 of Runov et al., 2006); blue diamonds show cases of the lowest ion drift velocity, $V_{i}<35 \mathrm{~km} \mathrm{~s}^{-1}$ $(8,9,10,19,21,29,30)$; and blue asterisks show cases $(20,24)$ of the lowest ion number density, $n_{i}<0.2 \mathrm{~cm}^{-3}$. Magenta circle shows the case 20 of extremely high velocity, $V_{i}=659 \mathrm{~km} \mathrm{~s}^{-1}$. All other "regular" cases are shown by red asterisks. The red line plots the fitting curve $y=1 /(0.045 x-0.01)$.

plasma, CS stability analysis and numerical simulations of magnetotail dynamics. So far these studies were restricted by purely symmetric background equilibria. In this paper we present the extension of the well-known family of exact kinetic Harris-Fadeev-Kan-Manankova solutions to the 2-D bent CS. This extension is really important, since the Earth dipole is tilted most of the time.

To validate the obtained analytic solution for bent CS we performed a comparison with the T96 model, used as a proxy of realistic averaged magnetospheric configuration. It is shown that the proposed model provides a reasonable approximation for the magnetotail CS in a wide range of dipole tilt angles and geomagnetic activity levels. Particularly, the parameters of the analytical model can always be adjusted to fit the behavior of the magnetic FTV with an accuracy of about $10 \%$ for all distances from 5 to $30 R_{\mathrm{E}}$ tailward. For short segments $\left(5 R_{\mathrm{E}}\right)$ of the CS, located beyond $15 R_{\mathrm{E}}$, the agreement may be improved up to $5 \%$ (except the case of the bent CS at quiet magnetospheric conditions). The agreement between analytical and empirical models is found to be better for the stretched magnetic configuration, i.e., for the pre-substorm conditions.

Notably, such a good agreement is obtained for the simplest three-parametric Kan-like model (Eqs. 7-9), where parameter $a$ controls the CS displacement from the equatorial plane, parameter $b_{0}$ controls magnetic field lines stretching, and parameter $\varphi$ specifies the CS bending. For further studies the more general model (Eqs. 11-16) can be considered, where additional parameters $n$ and $k$ provide the more accurate adjustment of the magnetoplasma quantities. Moreover, for sub-Alfvénic plasma, i.e., for the low-activity periods, all model parameters may be treated as time-dependent quantities (Wolf, 1983; Semenov et al., 2015). The time-dependent approach in such a modeling approach is not appropriate for the periods of explosive activity, such as storms and substorms, when BBFs with Alfvénic speed are produced.

Of course, the suggested analytical model is still far from universality. One significant limitation of this model is related to the isothermal constraint. This constraint may be released for four-component (two positive + two negative) plasma with bi-Maxwellian distribution functions for each particle species (Kan, 1973; Voronina and Kan, 1993). In such a case the condition (2) takes the form $V_{i k} / T_{i k}+$ $V_{e k} / T_{e k}=0$, where $k=\{1,2\}$. If two plasma components give zero contribution in the current velocity, $V_{i 2}-V_{e 2}=$ 0 , Eq. (1) stays valid for nonuniform plasma temperature (Voronina and Kan, 1993). The four-component-plasma model could be probably appropriate for magnetotail studies at high levels of geomagnetic activity. Indeed, in the quiet magnetotail the population of ions $\left\{\mathrm{O}^{+}, \mathrm{O}^{++}, \mathrm{He}^{++}\right\}$, penetrating from the ionosphere, is less than $1 \%$ (Lennartsson et al., 1986), and hence the approximation of "proton + electron" plasma is relevant. With the growth of geomagnetic activity, the $\mathrm{O}^{+}$contribution becomes essential during the main and recovery phases of intensive storm events. However, practical application of the nonisothermal model requires thorough studies, going beyond the scope of the present paper.

The constancy of the proton temperature is not reflected in observations (e.g., Kissinger et al., 2012; Wang et al., 2012), and hence the isothermal model may be considered as a first approximation only, although for some local analysis it seems to be rather suitable due to the small $(\sim 10$ $20 \%$ ) cross-cut variations of proton temperature, detected in observations of central-peaked current sheets (see Fig. 5 in Runov et al., 2006). In such sheets, inaccuracy of the constant-temperature estimate does not exceed the model inaccuracy in current density or CS width.

Other model limitations are the two-dimensionality and isotropy of the plasma pressure. Even with these limitations, the model stays appropriate for a wide class of problems, mentioned in the beginning of the current section. Particularly, we lay hopes that application of the presented model can stimulate investigations on the magnetotail CS stability to resolve the questions suggested by Kivelson and Hughes (1990): why can symmetric CS accumulate magnetic flux energy more effectively, and does the threshold of substorminitiating instability depend on degree of the CS bending?

Our findings are summarized as follows: 


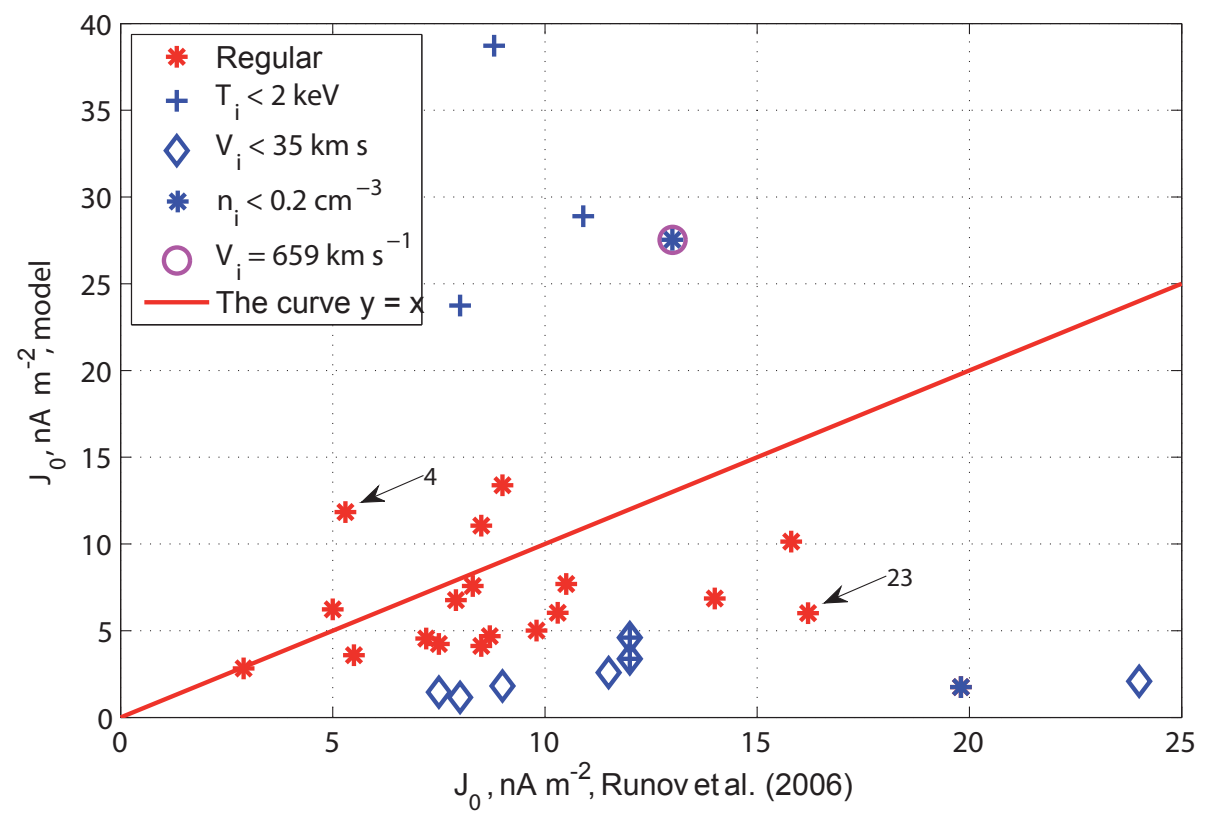

Figure 11. Typical current density $J_{0}$ of the analytical model vs. peaking perpendicular current density from Fig. 2 of Runov et al. (2006). Blue crosses show cases of the lowest ion temperature, $T_{i}<2 \mathrm{keV}(8,9,12,13,14$ in Table 1 of Runov et al. (2006)); blue diamonds show cases of the lowest ion drift velocity, $V_{i}<35 \mathrm{~km} \mathrm{~s}^{-1}(8,9,10,19,21,29,30)$; and blue asterisks show cases $(20,24)$ of the lowest ion number density, $n_{i}<0.2 \mathrm{~cm}^{-3}$. Magenta circle shows the case 20 of extremely high velocity, $V_{i}=659 \mathrm{~km} \mathrm{~s}^{-1}$. All other "regular" cases are shown by red asterisks. The red line plots $y=x$. Black arrows mark cases 4 and 23, demonstrating the largest (amongst red points) discrepancy of the observed and model values.

- An exact 2-D bent CS equilibrium, built by means of generalization of the Harris-Fadeev-Kan-Manankova family of symmetric solutions of the Vlasov-Maxwell equations, is considered. The examined model reproduces the effects, related to the Earth dipole tilt and CS bending. The further generalization releases degeneracy of the original model, which caused of the normal magnetic component to decrease too rapidly.

- Parameters of the asymmetric model may be adjusted to reproduce the realistic distribution of the magnetic flux tube volume at any level of geomagnetic activity; with enhancing activity the model relevance improves. The model-typical scales for CS width and current density match the corresponding parameters of the in situ registered single-peaked current sheets with medium values of number density, proton temperature and drift velocity; disagreement does not exceed a factor of 2 .

- The asymmetric solution does not contain any limitation for the tilt angle values, and hence the model is appropriate for any Earth-like magnetosphere with arbitrary dipole inclination.

- The obtained bent CS solution contains the X point, moving from infinity toward the dipole with the dipole tilt increase, staying still far beyond the lunar orbit for the Earth magnetotail realistic tilt angles. The location of the $\mathrm{X}$ point is much more effectively controlled by the new parameter $n$ of the generalized model (Eqs. 1116).

Data availability. No data sets were used in this article.

Competing interests. The authors declare that they have no conflict of interest.

Acknowledgements. This study has been supported by the Austrian Science Fund (FWF), P 27012-N27 and I 3506-N27, and by Russian Science Foundation (RSF) grant no. 18-47-05001. The authors thank Anna V. Egorova for her help with preparation of the images, and reviewers for their help in improving the paper.

The topical editor, Elias Roussos, thanks two anonymous referees for help in evaluating this paper.

\section{References}

Artemyev, A. V., Petrukovich, A. A., Zelenyi, L. M., Nakamura, R., Malova, H. V., and Popov, V. Y.: Thin embedded current sheets: Cluster observations of ion kinetic structure and analytical models, Ann. Geophys., 27, 4075-4087, https://doi.org/10.5194/angeo-27-4075-2009, 2009. 
Behannon, K. W.: Geometry of the geomagnetic tail, J. Geophys. Res., 75, 743-753, https://doi.org/10.1029/JA075i004p00743, 1970.

Behannon, K. W., and Ness, N. F.: Magnetic storms in the earth's magnetic tail, J. Geophys. Res., 71, 2327-2351, https://doi.org/10.1029/JZ071i009p02327, 1966.

Birn, J., Hesse, M., Schindler, K., and Zaharia, S.: Role of entropy in magnetotail dynamics, J. Geophys. Res., 114, A00D03, https://doi.org/10.1029/2008JA014015, 2009.

Burkhart, G. R., Drake, J. F., Dusenbery, P. B., and Speiser, T. W.: A particle model for magnetotail neutral sheet equilibria, J. Geophys. Res., 97, 13799-13815, https://doi.org/10.1029/92JA00495, 1992.

Cargill, P. G., Chen, J., and Holland, J. B.: One-dimensional hybrid simulations of current sheets in the quiet magnetotail, Geophys. Res. Lett., 21, 2251-2254, https://doi.org/10.1029/94GL01693, 1994.

Erkaev, N. V., Semenov, V. S., and Biernat, H. K.: Magnetic double-gradient instability and flapping waves in a current sheet, Phys. Rev. Lett., 99, 235003, https://doi.org/10.1103/PhysRevLett.99.235003, 2007.

Erkaev, N. V., Semenov, V. S., Kubyshkin, I. V., Kubyshkina, M. V., and Biernat, H. K.: MHD aspect of current sheet oscillations related to magnetic field gradients, Ann. Geophys., 27, 417-425, https://doi.org/10.5194/angeo-27-417-2009, 2009.

Fadeev, V. M., Kvartskhava, I. F., and Komarov, N. N.: Selffocusing of the local plasma currents, Nucl. Fusion, 5, 202-209, https://doi.org/10.1088/0029-5515/5/3/003, 1965 (in Russian).

Harris, E. G.: On a plasma sheath separating regions of oppositely directed magnetic field, Nuovo Cimento, 23, 115-121, https://doi.org/10.1007/BF02733547, 1962.

Hau, L. N., Wolf, R. A., Voigt, G. H., and Wu, C. C.: Steady state magnetic field configurations for the Earth's magnetotail, J. Geophys. Res. 94, 1303-1316, https://doi.org/10.1029/JA094iA02p01303, 1989.

Hoshino, M., Nishida, A., Mukai, T., Saito, Y., and Yamamoto, T.: Structure of plasma sheet in magnetotail: Double-peaked electric current sheet, J. Geophys. Res., 101, 24775-24786, https://doi.org/10.1029/96JA02313, 1996.

Kan, J. R.: On the structure of the magnetotail current sheet, J. Geophys. Res., 78, 3773-3781, https://doi.org/10.1029/JA078i019p03773, 1973.

Kissinger, J., McPherron, R. L., Hsu, T.-S., and Angelopoulos, V.: Diversion of plasma due to high pressure in the inner magnetosphere during steady magnetospheric convection, J. Geophys. Res., 117, A05206, https://doi.org/10.1029/2012JA017579, 2012.

Kivelson, M. G. and Hughes, W. J.: On the threshold for triggering substorms, Planet. Space Sci., 38, 211-220, https://doi.org/10.1016/0032-0633(90)90085-5, 1990.

Kubyshkina, M., Tsyganenko, N., Semenov, V., Kubyshkina, D., Partamies, N., and Gordeev, E.: Further evidence for the role of magnetotail current shape in substorm initiation, Earth Planets Space, 67, 139, https://doi.org/10.1186/s40623-015-0304-1, 2015.

Lennartsson, W. and Shelley, E. G.: Survey of 0.1- to 16-keV/e plasma sheet ion composition, J. Geophys. Res., 91, 3061-3076, https://doi.org/10.1029/JA091iA03p03061, 1986.
Manankova, A. V., Pudovkin, M. I., and Runov, A. V.: Stationary configurations of the two-dimensional current-carrying plasma sheet: Exact solutions, Geomagn. Aeronomy+, 40, 430-438, 2000 .

Mihalov, J. D., Colburn, D. S., Currie, R. G., and Sonett, C. P.: Configuration and reconnection of the geomagnetic tail, J. Geophys. Res., 73, 943-959, https://doi.org/10.1029/JA073i003p00943, 1968.

Nakamura, R., Baumjohann, W., Runov, A., and Asano, Y.: Thin Current Sheets in the Magnetotail Observed by Cluster, Space Sci. Rev., 122, 29-38, https://doi.org/10.1007/s11214-006-62191, 2006.

Nowada, M., Shue, J.-H., and Russel, C. T.: Effects of dipole tilt angle on geomagnetic activity, Planet. Space Sci., 57, 1254-1259, https://doi.org/10.1016/j.pss.2009.04.007, 2009.

Panov, E. V., Nakamura, R., Baumjohann, W., Kubyshkina, M. G., Artemyev, A. V., Sergeev, V. A., Petrukovich, A. A., Angelopoulos, V., Glassmeier, K.-H., McFadden, J. P., and Larson, D.: Kinetic ballooning/interchange instability in a bent plasma sheet, J. Geophys. Res., 117, A06228, https://doi.org/10.1029/2011JA017496, 2012.

Panov, E. V., Baumjohann, W., Wolf, R. A., Nakamura, R., Angelopoulos, V., Weygand, J. M., and Kubyshkina, M. V.: Magnetotail energy dissipation during an auroral substorm, Nat. Phys., 12, 1158-1163, https://doi.org/10.1038/nphys3879, 2016.

Partamies, N., Pulkkinen, T. I., McPherron, R. L., McWilliams, K., Bryant, C. R., Tanskanen, E., Singer, H. J., Reeves, G. D., and Thomsen, M. F.: Different magnetospheric modes: solar wind driving and coupling efficiency, Ann. Geophys., 27, 4281-4291, https://doi.org/10.5194/angeo-27-4281-2009, 2009.

Petrukovich, A., Artemyev, A., Vasko, I., Nakamura, R., and Zelenyi, L.: Current Sheets in the Earth Magnetotail: Plasma and Magnetic Field Structure with Cluster Project Observations, Space Sci. Rev., 188(1-4), 311-337, https://doi.org/10.1007/s11214-014-0126-7, 2015.

Pritchett, P. L. and Coroniti, F. V.: Formation and stability of the self-consistent one-dimensional current sheet, J. Geophys. Res., 97, 16773-16787, https://doi.org/10.1029/92JA01550, 1992.

Pritchett, P. L. and Coroniti, F. V.: A kinetic ballooning/interchange instability in the magnetotail, J. Geophys. Res., 115, A06301, https://doi.org/10.1029/2009JA014752, 2010.

Runov, A., Sergeev, V. A., Nakamura, R., Baumjohann, W., Apatenkov, S., Asano, Y., Takada, T., Volwerk, M., Vörös, Z., Zhang, T. L., Sauvaud, J.-A., Réme, H., and Balogh, A.: Local structure of the magnetotail current sheet: 2001 Cluster observations, Ann. Geophys., 24, 247-262, 2006.

Schindler, K.: A self-consistent theory of the tail of the magnetosphere, in: Earth's Magnetospheric Processes, vol. 32, edited by: McComas, B. M., D. Reidel, Dordrecht, pp. 200-209, 1972.

Schindler, K. and Birn, J.: Models of two-dimensional embedded thin current sheets from Vlasov theory, J. Geophys. Res., 107, 1193, https://doi.org/10.1029/2001JA000304, 2002.

Semenov, V. S., Kubyshkina, D. I., Kubyshkina, M. V., Kubyshkin, I. V., and Partamies, N.: On the correlation between the fast solar wind flow changes and substorm occurrence, Geophys. Res. Lett., 42, 5117-5124, https://doi.org/10.1002/2015GL064806, 2015.

Sergeev, V. A., Chernyaev, I. A., Angelopoulos, V., Runov, A. V., and Nakamura, R.: Stopping flow bursts and their role in the gen- 
eration of the substorm current wedge, Geophys. Res. Lett., 41, 1106-1112, https://doi.org/10.1002/2014GL059309, 2014.

Sitnov, M. I. and Merkin, V. G.: Generalized magnetotail equilibria: Effects of the dipole field, thin current sheets, and magnetic flux accumulation, J. Geophys. Res., 121, 7664-7683, https://doi.org/10.1002/2016JA023001, 2016.

Tsyganenko, N. A.: Modeling the Earth's magnetospheric magnetic field confined within a realistic magnetopause, J. Geophys. Res., 100, 5599-5612, https://doi.org/10.1029/94JA03193, 1995.

Vasko, I. Y., Artemyev, A. V., Popov, V. Y., and Malova, H. V.: Kinetic models of two-dimensional plane and axially symmetric current sheets: Group theory approach, Phys. Plasmas, 20, 022110, https://doi.org/10.1063/1.4792263, 2013.

Vinogradov, A. A., Vasko, I. Y., Artemyev, A. V., Yushkov, E. V., Petrukovich, A. A., and Zelenyi, L. M.: Kinetic models of magnetic flux ropes observed in the earth magnetosphere, Phys. Plasmas, 23, 072901, https://doi.org/10.1063/1.4958319, 2016.

Voronina, V. A. and Kan, J. R.: A kinetic model of the plasma sheet: isotropic nonuniform plasma temperature, J. Geophys. Res., 98, 13395-13402, https://doi.org/10.1029/93JA00959, 1993.

Walker, G. W.: Some problems illustrating the forms of nebulae, P. Roy. Soc. Lond. A Mat., 91, 410-420, https://doi.org/10.1098/rspa.1915.0032, 1915.

Wang, C.-P. and Lyons, L. R.: Midnight radial profiles of the quiet and growth-phase plasma sheet: The Geotail observations, J. Geophys. Res., 109, A12201, https://doi.org/10.1029/2004JA010590, 2004.
Wang, C.-P., Gkioulidou, M., Lyons, L. R., and Angelopoulos, V.: Spatial distributions of the ion to electron temperature ratio in the magnetosheath and plasma sheet, J. Geophys. Res., 117, A08215, https://doi.org/10.1029/2012JA017658, 2012.

Wolf, R. A.: The quasi-static (slow-flow) region of the magnetosphere, in: Solar-Terrestrial Physics, vol. 104, edited by: Carovillano, R. L and Forbes, J. M., Springer, Dordrecht, https://doi.org/10.1007/978-94-009-7194-3_14, 303-368, 1983.

Yoon, P. H. and Lui, A. T. Y.: Lower-hybrid-drift and modifiedtwo-stream instabilities in current sheet equilibrium, J. Geophys Res., 110, A02210, https://doi.org/10.1029/2003JA010180, 2004.

Yoon, P. H. and Lui, A. T. Y.: A class of exact two-dimensional kinetic current sheet equilibria, J. Geophys. Res., 110, A01202, https://doi.org/10.1029/2003JA010308, 2005.

Yue, C., Wang, C.-P., Zaharia, S. G., Xing, X., and Lyons, L.: Empirical modeling of plasma sheet pressure and threedimensional force-balanced magnetospheric magnetic field structure: 2. Modeling, J. Geophys. Res., 118, 6166-6175, https://doi.org/10.1002/2013JA018943, 2013.

Zaharia, S. and Cheng, C. Z.: Can an isotropic plasma pressure distribution be in force balance with the T96 model field?, J. Geophys. Res., 108, 1412, https://doi.org/10.1029/2002JA009501, 2003. 